\section{Chryseobacterium/Elizabethkingia species infections in Saudi Arabia}

\author{
Abdulaziz M. Alyami, MSN, Naoufel M. Kaabia, MD, \\ Marzouq A. AlQasim, BSN, Fahad S. Al Doghaim, MID, \\ Lulu B. Albehlal, BA, Medina A. Ahmed, MD, PhD, \\ Amal Y. Al Aidaroos, MD, Abdurabman Al Odayani, MD.
}

\section{ABSTRACT}

Objectives: To describe the epidemiological, clinical, and outcome data of patients infected or colonized with Chryseobacterium/Elizabethkingia spp including antibiotic susceptibility patterns.

Methods: This retrospective study was conducted at Prince Sultan Military Medical City, Riyadh, Saudi Arabia. All patients infected or colonized by Chryseobacterium /Elizabethkingia spp who were admitted between June 2013 and May 2019 were included. Data were extracted from patient electronic medical records.

Results: We enrolled 27 patients (13 males and 14 females) with a mean age of 35.6 years. Chryseobacterium/ Elizabethkingia spp were isolated from blood cultures $(\mathrm{n}=13,48 \%)$ and tracheal aspirations $(\mathrm{n}=11,41 \%)$. The most frequent species isolated was Elizabethkingia meningoseptica $(\mathrm{n}=22)$. Although 6 patients were considered colonized, the remaining 21 patients presented with ventilator associated pneumonia $(n=9)$, central line associated bloodstream infection $(n=4)$, septic shock $(n=4)$, or isolated bacteremia $(n=4)$. In 25 cases the infections were health-care related. Three patients (11\%) died within 28 days. Twenty-six isolates (96.5\%) were resistant to carbapenems. Moxifloxacin and cotrimoxazole were the most active antibiotics.

Conclusion: Chryseobacterium/Elizabethkingia spp infection is rare, but can be responsible for severe hospital acquired infections. Cotrimoxazole and fluoroquinolone are the most effective antibiotic treatments.

Keywords: Chryseobacterium, Elizabethkingia, infection, antibiotic resistance, mortality

Saudi Med J 2020; Vol. 41 (3): 309-313 doi: 10.15537/smj.2020.3.24985

Cryseobacterium/Elizabethkingia species
are rod-shaped Gram-negative bacteria. Chryseobacterium genus consist of 6 species that were previously classified as Flavobacterium. Elizabethkingia meningoseptica and Chryseobacterium indologenes are
2 species commonly isolated from clinical specimens; Elizabethkingia meningoseptica is reported as the most virulence member of the genus. This ubiquitous bacterium inhabits natural and hospital environments. ${ }^{1}$ It has been reported to colonizing hospital water supplies, sink basins, saline solution and taps, leading to increase the risk of infection inside hospital environments. In addition to that isolation of the bacteria from medical devices such as feeding tubes, respirators, syringes and arterial catheters has been also documented. Infections with Chryseobacterium/Elizabethkingia spp are usually associated with indwelling devices and often affect neonates and immunocompromised patients. ${ }^{2,3}$ There is limited data about antimicrobial susceptibility testing for Chryseobacterium/Elizabethkingia spp because the isolation of these pathogens from clinical specimens are uncommon. In addition, susceptibility testing results vary depending on the method used. Chryseobacterium/ Elizabethkingia spp are intrinsically resistant to a wide range of different antibiotics. ${ }^{4}$ However, the organisms have been shown to have sensitivity to some fluoroquinolones. ${ }^{5}$ Thus, the aim of the current study is to describe the clinical, epidemiology, and outcome data of patients colonized and infected with Chryseobacterium/Elizabethkingia spp, as well as outline the antibiotic susceptibility patterns.

Methods. All patients infected or colonized by Chryseobacterium/Elizabethkingia at Prince Sultan Military Medical City (PSMMC), Riyadh, Saudi Arabia during the 6-year period between June 2013 and May 2019 were included in this study. Demographic characteristics, clinical data, outcome of patients and antimicrobial susceptibility of bacterial isolates were extracted from electronic medical records as well as infection control surveillance data. Identification of Chryseobacterium/Elizabethkingia spp was relied on conventional culture techniques of clinical samples. The isolated colonies were further identified using manual or automated phenotypic methods. These included manual biochemical reactions using the API $20 \mathrm{NE}$ identification system (bioMérieux, Marcy l'Etoile, France) for non-fastidious, non-enteric Gram-negative rods and the automated MicroScan WalkAway system (Beckman Coulter, South Kraemer Boulevard Brea, California) using the conventional dried Gram-negative identification panel Neg Breakpoint Combo 50. For all bacteremic patients and patients admitted to the ICU both methods were used and discrepancies were sent for verification of identification by MALDI-TOF MS system. Antimicrobial susceptibility testing of the isolates was carried out according to the guidelines of 
the Clinical and Laboratory Standards Institute (CLSI) ${ }^{4}$ to determine either the breakpoint or the minimum inhibitory concentration (MIC) of all antibiotics. Breakpoints were identified using the MicroScan WalkAway Neg Breakpoint Combo-50 panel and MIC was determined using E test strips (bioMérieux, Marcy l'Etoile, France). Statistical Package for Social Sciences ${ }^{\circ}$ Version 20 (Armonk, NY: IBM Corp.) was used for data analysis.

Results. Twenty-seven patients were identified as infected or colonized by Chryseobacterium/
Elizabethkingia spp. These infections represented 0.32\% of all reported multi-drug resistant organism (MDRO) cases (8420 total) during the same period. No cluster cases or outbreaks were noted. The most frequent species isolated were Elizabethkingia meningoseptica $(\mathrm{n}=22)$ and $C$. indologenes $(\mathrm{n}=4)$. The source of the infection was within the hospital for 25 cases (92.5\%), with the majority acquired in ICUs $(n=20)$. The average time between admission and positive culture was 240.8 days. Risk factors for MDROs and mortality rate are summarized in Table 1. Regarding the susceptibility of Chryseobacterium/Elizabethkingia spp to antibiotics,

Table 1 - Demographical, epidemiological, clinical data and outcomes of patients with positive culture for Chryseobacterium/ Elizabethkingia $\operatorname{spp}$ at Prince Sultan Military Medical City, Riyadh, Saudi Arabia.

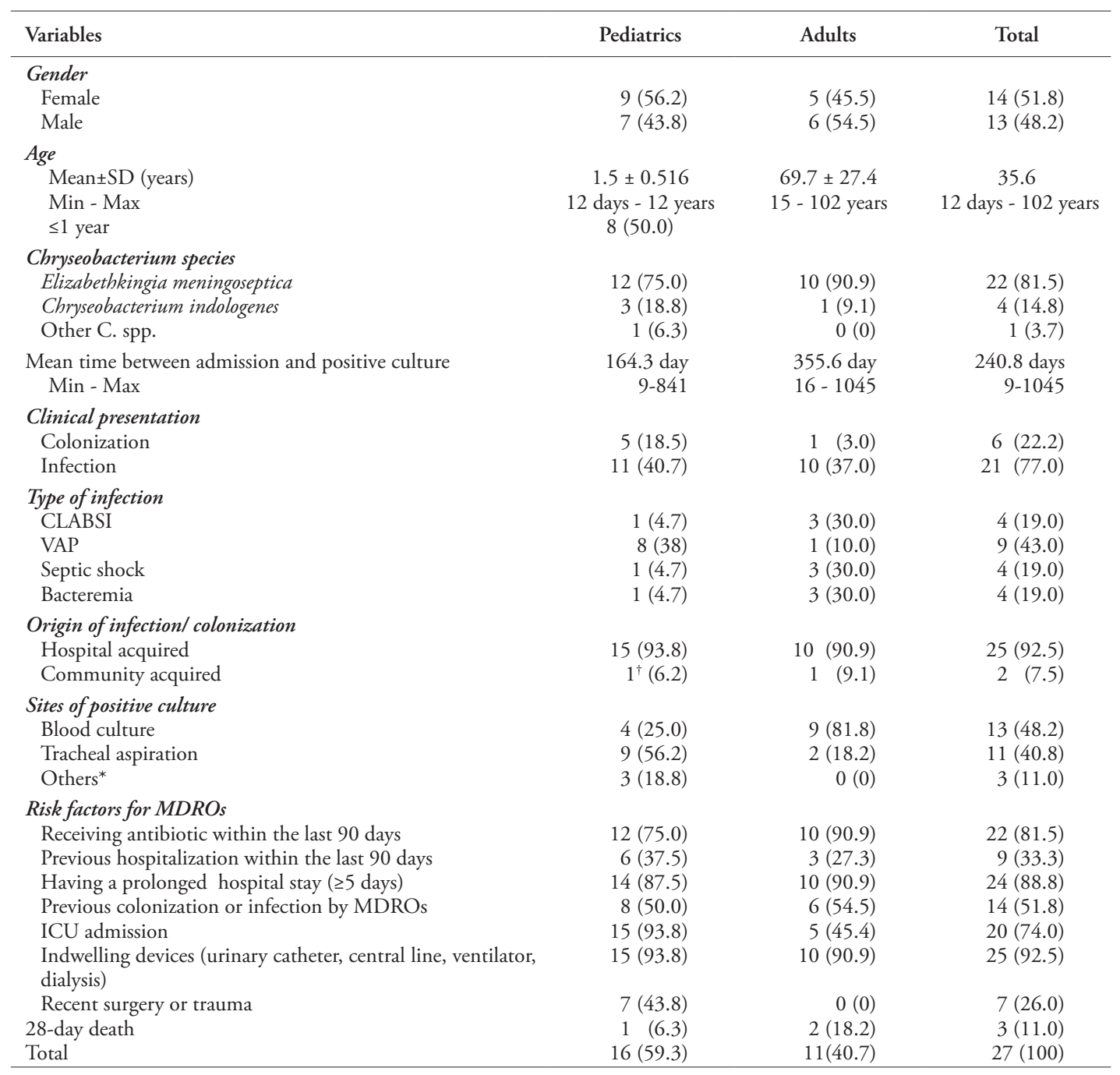

"eye secretion, biliary drain, and nasopharyngeal aspirate, "hemodialysis patient, CLABSI: central line associated blood stream infection, VAP: ventilator associated pneumonia, MDROs: multi-drug resistant organisms, E: Elizabethkingia, C: Chryseobacterium 


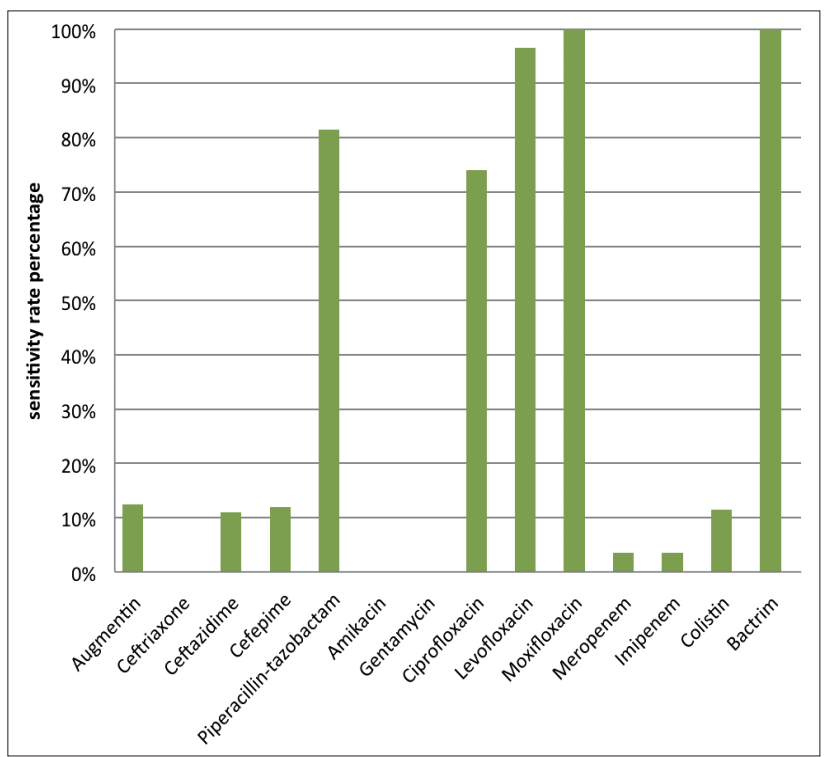

Figure 1 - Chryseobacterium/Elizabethkingia spp sensitivity rate to selected antibiotics at Prince Sultan Military Medical City, Riyadh, Saudi Arabia

Moxifloxacin and cotrimoxazole were the most active antibiotics. Vancomycin was tested for only one isolate and showed resistance with minimum inhibitor concentration (MIC) E-test $=12 \mathrm{mg}$. Antibiotic sensitivity rates for our series are shown in Figure 1.

Discussion. Although, Chryseobacterium/ Elizabethkingia spp are generally low virulence, they may occasionally be involved in severe infections. Because infections with these pathogens are relatively rare, limited studies are available. During a worldwide surveillance including 119 sentinel hospitals and laboratories between 1997 to 2001 only 50 Chryseobacterium/ Elizabethkingia spp were isolated out of 155,811 clinical bacterial isolates from several sites. Only $0.10 \%$ of Chryseobacterium/Elizabethkingia spp were isolated from respiratory tract and $0.03 \%$ were isolated from blood. ${ }^{6}$ A previous study was performed at King Khalid Hospital, south of Saudi Arabia showed that among 125 non-duplicating, non-fermenting, Gram-negative bacilli, nosocomial strains that were isolated, only 5 cases were Chryseobacterium indologenes. ${ }^{7}$ At PSMMC, a teaching hospital with a 1350 bed capacity, 27

Disclosure. Authors have no conflict of interests, and the work was not supported or funded by any drug company. patients presented with infection or were colonized by Chryseobacterium/Elizabethkingia spp. This represented $0.32 \%$ of all MDROs reported to the infection control department during a 6-year span. The rarity of this pathogen as a cause of infection is probably related to its reduced degree of pathogenicity. Some studies showed that the immune system of healthy human can rule out the Chryseobacterium/Elizabethkingia spp when introduced into the bloodstream or respiratory tract. ${ }^{8}$ In this study, many patients cleared their bacteremia despite the inappropriate antibiotic regimens prescribed for them. In addition, one patient on hemodialysis had persistent Elizabethkingia meningoseptica bacteremia for 6 days without any antibiotic treatment, yet remained clinically stable apart from spikes of fever. This study includes a significant number of Chryseobacterium/ Elizabethkingia spp isolated from clinical specimens in Arabian Peninsula countries. Infections are commonly nosocomial and often associated with indwelling devices. Patients often infected with Chryseobacterium/ Elizabethkingia spp when they usually exposed to contaminated medical devices such as catheters, humidifiers, feeding tubes, respirators and syringes. ${ }^{2}$ Long term use of antibiotics is also considered as important risk factor. ${ }^{6}$ In our study, $92.5 \%$ of cases were hospital-acquired, with a long mean time span between admission and the date of first positive culture (240.8 days). The majority of patients $(25 / 27)$ had at least one device. These were predominantly endotracheal tubes in 20 patients. Previous studies ${ }^{2,3}$ have reported that Chryseobacterium/Elizabethkingia spp can grow in water supplies treated with chlorine, and often colonizing sink basins, saline solution and taps, leading to increase the risk of infection inside hospital environments. In addition to that isolation of the bacteria from medical devices such as feeding tubes, respirators, syringes and arterial catheters and surgical implanted devices has been also documented. Although Chryseobacterium/ Elizabethkingia spp infections are known to be noted on small outbreaks, especially among neonates, in our study all cases were sporadic and occurred in different hospital areas (mostly ICUs), and majority of our patients were adults or infants. Only one neonatal case was noted.

Among the species of Chryseobacterium/ Elizabethkingia, Chryseobacterium indologenes is the most well-known bacterium and is most commonly isolated from clinical specimens. It is generally isolated in immunocompromised patients and therefore its clinical significance has been questioned. ${ }^{3,9}$ In contrast, Elizabethkingia meningoseptica is the most pathogenic species, and has been found to be responsible for many human infections and outbreaks. ${ }^{10,11}$ According 
to SENTRY Antimicrobial Surveillance Program contacted between 1997 to 2001, 50 Chryseobacterium /Elizabethkingia spp were isolated, mostly among elderly patients. The majority of the isolates was Elizabethkingia meningoseptica (E. meningoseptica) (48\%), following by $C$. indologenes (40\%), and C. gleum (4\%). ${ }^{6}$ In the current study, the majority of species isolated were E. meningoseptica $(\mathrm{n}=22)$, and most of these involved infant and elderly patients; we did not note any meningitis cases among neonates or infants. Most C. indologenes cases were reported in adult patients; few were reported in children. ${ }^{13}$ In our series, among 4 cases of $C$. indologenes, 3 were noted in children. Adults with Chryseobacterium/Elizabethkingia spp infections may present with pneumonia, septicemia, meningitis, endocarditis, or infections after surgeries or burn. Gunnarsson et $\mathrm{al}^{13}$ reported a case of septic arthritis due to $E$. meningoseptica in an immunocompetent patient, despite the fact that infections usually occur in immunosuppressed patients.

Many studies showed that Chryseobacterium/ Elizabethkingia spp are resistance to many different types of antimicrobials especially antibiotics used to treat Gram-negative bacteria. However, they showed susceptibility to agents used to treat Gram-positive infections. Chryseobacterium/Elizabethkingia spp have shown resistance to erythromycin, chloramphenicol, erythromycin, clindamycin, tetracycline and teicoplanin, and susceptibility to some fluoroquinolone antibiotics. ${ }^{4,14,15}$ Rifampin is shown activity in vitro against Chryseobacterium/Elizabethkingia spp and usually used as combination with vancomycin to treat the infection. ${ }^{6}$ However, the use of vancomycin to treat Chryseobacterium/Elizabethkingia spp infection is limited. ${ }^{4}$ Thus, there is no evidence base to define the optimal treatment for Chryseobacterium/Elizabethkingia $s p p$ infections and the choice of antibiotics has to be based on antimicrobial susceptibility testing results. According to SENTRY Antimicrobial Surveillance Program contacted between 1997 to 2001, levofloxacin, garenoxacin and gatifloxacin showed high antimicrobial activity against Chryseobacterium/Elizabethkingia spp. In addition to that rifampin, sulfamethoxazole, piperacillintazobactam and ciprofloxacin, also exhibited good activity, and aminoglycosides and carbapenems showed poor susceptibility with $14 \%$ and $12 \%$ respectably. The vancomycin reported to show poor activity. ${ }^{6}$ In our series, moxifloxacin and cotrimoxazole were the most active antibiotics. Vancomycin was tested for only one isolate and showed resistance with MIC E-test $=12 \mathrm{mg}$. Rifampicin was not tested.
Study limitations. This was a retrospective case series study, and the accuracy and availability of data might have concealed potential risk factors that were not documented in the medical records.

In conclusion, Chryseobacterium/Elizabethkingia spp at PSMMC are rare emergent pathogens responsible for health care associated infections. These are mainly seen in patients with long hospital stays and/or indwelling devices (especially endotracheal tubes), and in patients who received antibiotics within the last 3 months.

Elizabethkingia meningoseptica was the most frequent species isolated, and the majority of patients infected by this bacterium presented with blood stream infections and/or ventilator associated pneumonia. The most effective antibiotics against this organism were cotrimoxazole and fluoroquinolone.

Received 18th September 2019. Accepted 6th January 2020.

From the Department of Infection Control and Prevention (Alyami, Kaabia, AlQasim, Al Doghaim, Al Aidaroos, Al Odayani), Central Laboratory and Blood Bank (Ahmed), Scientific Research Center (Albehlal), Prince Sultan Military Medical City, Riyadh, Kingdom of Saudi Arabia.

Address correspondence and reprints request to: Dr. Naoufel M. Kaabia, Infection Control and Prevention Department, Prince Sultan Military Medical City, Riyadh, Kingdom of Saudi Arabia.E-mail: nkaabia@psmmc.med.sa ORCID ID: https://orcid.org/0000-0002-1338-0546

\section{References}

1. Vandamme P, Bernardet J-F, Segers P, Kersters K, Holmes B. New perspectives in the classification of the flavobacteria: Description of Chryseobacterium gen. rev Int J Syst Bacteriol 1994; 44: 827-831.

2. Rishika Mehta, Ashish Pathak. Emerging Chryseobacterium indologenes infection in Indian neonatal intensive care units: A case report. Antibiotics 2018; 7: pii: E109.

3. Nulens E, B Bussels, A Bols, B Gordts, and H W Van Landuyt. Recurrent bacteremia by Chryseobacterium indologenes in an oncology patient with a totally implanted intravenous device. Clin Microbiol Infect 2001; 7: 391-393.

4. Mirza HC, Tuncer Ö, Ölmez S, Şener B, Tuğcu GD, Özçelik U,et al. clinical Strains of Chryseobacterium and Elizabethkingia $s p p$ isolated from pediatric patients in a university hospital: Performance of MALDI-TOF MS-based identification, antimicrobial susceptibilities, and baseline patient characteristics. Microb Drug Resist 2018; 24: 816-821.

5. Chen FL, Wang GC, Teng SO, Ou TY, Yu FL, Lee WS. Clinical and epidemiological features of Chryseobacterium indologenes infections: Analysis of 215 cases. J Microbiol Immunol Infect 2013; 46: 425-432.

6. Kirby JT, Sader HS, Walsh TR, Jones RN. Antimicrobial susceptibility and epidemiology of a worldwide collection of Chryseobacterium spp: report from the SENTRY Antimicrobial Surveillance Program (1997-2001). J Clin Microbiol 2004; 42: 445-448.

7. Asaad AM, Al-Ayed MS, Qureshi MA. Emergence of unusual nonfermenting Gram-negative nosocomial pathogens in a Saudi hospital. Jpn J Infect Dis 2013; 66: 507-511. 
8. Esposito S, Russo E, De Simone G, Gioia R, Noviello S, Vitolo M, et al. Transient bacteraemia due to Chryseobacterium indologenes in an immunocompetent patient: a case report and literature review. J Chemother 2015; 27: 324-329.

9. Osamu Imataki, Makiko Uemura. Chryseobacterium indologenes, a possible emergent organism resistant to carbapenem antimicrobials after stem cell transplantation. Clin Case Rep 2017; 5: 22-25.

10. Lin JN, Lai CH, Yang CH, Huang YH. Elizabethkingia Infections in Humans: From Genomics to Clinics. Microorganisms 2019; 7: pii: E295.

11. Jean SS, Lee WS, Chen FL, Ou TY, Hsueh PR. Elizabethkingia meningoseptica: an important emerging pathogen causing healthcare-associated infections. J Hosp Infect 2014; 86: 244-249.
12. Aykac K, Ozsurekci Y, Tuncer O, Sancak B, Cengiz AB, Kara A, Ceyhan M. Six cases during 2012-2015 and literature review of Chryseobacterium indologenes infections in pediatric patients. Can J Microbiol 2016; 62: 812-819.

13. Gunnarsson G, Baldursson H, Hilmarsdottir I. Septic arthritis caused by Chryseobacterium meningosepticum in an immunocompetent male. Scand J Infect Dis 2002; 34: 299-300.

14. Han MS, Kim H, Lee Y, Kim M, Ku NS, Choi JY, et al. Relative prevalence and antimicrobial susceptibility of clinical isolates of Elizabethkingia species based on $16 \mathrm{~S}$ rRNA gene sequencing. $J$ Clin Microbiol 2016; 28; 55: 274-280.

15. Rolston KV, Vaziri I, Frisbee-Hume S, Streeter H, LeBlanc B. In vitro antimicrobial activity of gatifloxacin compared with other quinolones against clinical isolates from cancer patients. Chemotherapy 2004; 50: 214-220. 